\title{
How Safe Are Neurosurgical Patients during Intrahospital Transportation?
}

\author{
Avondeep Dhaliwal ${ }^{1}$ Meenakshi Agnihotri ${ }^{1}$ Sandeep Mohindra ${ }^{2}$ \\ ${ }^{1}$ National Institute of Nursing Education, Postgraduate Institute of \\ Medical Education and Research, Chandigarh, India \\ 2 Department of Neurosurgery, Postgraduate Institute of Medical \\ Education and Research, Chandigarh, India \\ Address for correspondence Avondeep Dhaliwal, MSc, Division of \\ Neurology and Neurosurgery Nursing, National Institute of Nursing \\ Education, Postgraduate Institute of Medical Education and Research, \\ Chandigarh, India (e-mail: avondhaliwal89@gmail.com).
}

Indian J Neurosurg 2015;4:26-30.

\begin{abstract}
Background Transporting critically sick neurosurgical patients from the intensive care environment for diagnostic procedures has become increasingly common. The occurrence of physiologic compromise and technical mishaps has been recognized. Objective To assess predeparture, in-transit, and arrival procedures and documentation during intrahospital transport of critically sick neurosurgical patients.

Keywords

- intrahospital transport

- critically sick neurosurgical patients

- checklist Materials and Methods In a study of consecutive 30 patients, the transfer/shifting details were prospectively analyzed.

Results The present study did not document any untoward incident and fixed protocol/checklist was followed for all cases/transfers.

Conclusion The study concluded that safe intrahospital transport of critically sick neurosurgical patients can be ensured by following a systemic checklist. So, there is a need to utilize transportation checklist for better outcome of neurosurgical patients.
\end{abstract}

\section{Introduction}

The safest place for critically sick neurosurgical patients remains the intensive care unit (ICU). ${ }^{1}$ However, many a times, transportation of these patients becomes mandatory because of diagnostic and therapeutic purposes. Transportation exposes these patients to potential hemodynamic instability, increased risk of complications, morbidity, and mortality. ${ }^{2}$ The incidence of hemodynamic instability in such cases is as high as $68 \% .^{3}$ Transportation has been associated with mishaps ranging from technical to severe life-threatening events. ${ }^{4}$

In the present study, the investigators assessed predeparture, in-transit, and arrival procedures and documentation during intrahospital transport of critically sick neurosurgical patients.

\section{Materials and Methods}

The study was conducted using quantitative, descriptive prospective survey approach. A total 30 consecutive patients of Intensive Care Unit, PGIMER, Chandigarh (tertiary care hospital, with bed strength over 1,780), formed the part of study. The study was undertaken from July 2013 to September 2013, for a period of 12 weeks. Observations were made and recorded on the respective checklists, maintaining the confidentiality of data. A checklist was developed for collection of data from study subjects. Reliability of the checklist was established, with a reliability coefficient (Cronbach alpha) of 0.802 .

\section{Results}

-Table 1 shows that of the total 30 patients, 17 (56.7\%) patients required transportation on account of a radiological investigation only, which was CT scan in our setup. Further, 9 (30\%) patients required shifting while getting into postoperative convalesce period and were shifted into less dependent general ward or rehabilitation areas. The time taken for transportation was maximum while patients were undergoing CT scan (25.18 minutes). Shifting of patients published online March 21, 2015
DOI http://dx.doi.org/ $10.1055 / \mathrm{s}-0035-1549066$. ISSN 2277-954X. (c) 2015 Neurological Surgeons' Society of India
License terms

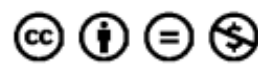


Table 1 Purpose and time consumption of patient transfer $(N=30)$

\begin{tabular}{|l|l|l|l|}
\hline From & For & $\boldsymbol{n}(\%)$ & Mean \pm SD (min) \\
\hline Neurosurgery ICU & CT scan & $17(56.7)$ & $25.18 \pm 4.98$ \\
\hline Neurosurgery ICU & Interward (general ward) & $9(30.0)$ & $17.00 \pm 7.39$ \\
\hline Neurosurgery ICU & Interward (personal ward) & $2(6.7)$ & $25.00 \pm 7.07$ \\
\hline Neurosurgery ICU & Inter ward (rehabilitation ward) & $1(3.3)$ & 13.00 \\
\hline Neurosurgery ICU & Interward (specialty ward) & $1(3.3)$ & 5.00 \\
\hline
\end{tabular}

Table 2 Changes in biophysiological parameters before and after transport $(N=30)$

\begin{tabular}{|l|l|l|l|}
\hline \multirow{2}{*}{ Variables } & Before transport & After arrival at destination & \multirow{2}{*}{-value } \\
\cline { 2 - 4 } & Mean \pm SD & Mean \pm SD & 0.254 \\
\hline Heart rate & $92.10 \pm 15.302$ & $92.70 \pm 14.751$ & - \\
\hline Temperature & $37.25 \pm 0.497$ & $37.25 \pm 0.497$ & $0.028^{\text {a }}$ \\
\hline Respiration rate & $20.80 \pm 2.27$ & $21.57 \pm 2.16$ & 0.573 \\
\hline Oxygen saturation & $99.93 \pm 0.254$ & $99.97 \pm 0.183$ & 0.273 \\
\hline Systolic blood pressure & $137.60 \pm 16.915$ & $138.20 \pm 17.860$ & 0.263 \\
\hline Diastolic blood pressure & $82.80 \pm 11.183$ & $81.80 \pm 10.845$ & - \\
\hline Glasgow Coma Scale & $12.73 \pm 2.959$ & $12.73 \pm 2.959$ & \\
\hline
\end{tabular}

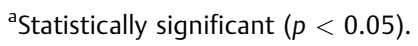

from ICU to personal ward involved nearly the same amount of time of 25 minutes.

The changes in biophysiological parameters were recorded (see - Table 2). Maximal changes were noted in respiratory rate, which was statistically significant, while heart rate, temperature, oxygen saturation, systolic blood pressure, diastolic blood pressure, and coma scale parameters also showed changes but were insignificant.

\section{Predeparture Procedures}

-Table 3 highlights communication regarding patient transport; both departing and receiving teams established the communication, but the content of information exchange was also noted. Of the 30 patients, the clinical status information was communicated to receiving team in 25 (83.3\%) cases only. The exact time of departure and expected time of receiving the patient were communicated in only 7 (23.3\%) patients. The treating clinician accompanied the patient in 27 (90\%) transports, while nursing personnel accompanied in 12 (40\%) cases. Patients' relative and hospital attendant accompanied in all transports.

- Table 4 shows that all patient transports were undertaken on trolleys, which were able to enter lifts and pass through all doorways en route. However, wheel

Table 3 Predeparture coordination and communication and accompanying staff $(N=30)$

\begin{tabular}{|c|c|c|c|c|}
\hline SI. no. & Content & Done, $n(\%)$ & Not done, $n(\%)$ & Not Applicable, $n$ \\
\hline \multirow[t]{4}{*}{ a. } & \multicolumn{4}{|c|}{ Before transport coordination and communication } \\
\hline & Receiving staff informed & $30(100)$ & - & - \\
\hline & Patient status explained to receiving staff & $25(83.3)$ & $5(16.7)$ & - \\
\hline & Tentative time of arrival informed & $7(23.3)$ & $23(76.7)$ & - \\
\hline \multirow[t]{5}{*}{ b. } & \multicolumn{4}{|l|}{ Accompanying staff: } \\
\hline & Treating clinician & $27(90)$ & $3(10)$ & - \\
\hline & Nursing personnel & $12(40)$ & $18(60)$ & - \\
\hline & Patient relative & $30(100)$ & - & - \\
\hline & Hospital attendant & $30(100)$ & - & - \\
\hline
\end{tabular}


Table 4 Accompanying equipment for intrahospital transportation $(N=30)$

\begin{tabular}{|c|c|c|c|c|}
\hline Sl. no. & Content & Done, $n(\%)$ & Not done, $n(\%)$ & Not applicable, $n$ \\
\hline 1. & Available trolley with functioning wheels & $23(76.7)$ & $7(23.3)$ & - \\
\hline 2. & $\begin{array}{l}\text { Trolley must be able to enter lifts and pass } \\
\text { through all doorways en route }\end{array}$ & $30(100)$ & - & - \\
\hline 3. & $\begin{array}{l}\text { Intubation kit } \\
\text { (oral/nasal airways, various size of ET } \\
\text { tube, complete suction set-up, laryngoscope } \\
\text { with blades, Assisted Mechanical Breathing } \\
\text { Unit, Magill forceps, a pair of sterile } \\
\text { scissors, Xylocaine jelly, and gloves) }\end{array}$ & $19(70.3 \%)$ & $11(36.7 \%)$ & - \\
\hline 4. & Full oxygen cylinder with flow meter & $27(100)$ & - & 3 \\
\hline 5. & Portable ventilator & - & $5(100)$ & 25 \\
\hline 6. & Portable cardiac monitor & $5(20)$ & $20(80)$ & 5 \\
\hline 7. & BP cuff & $17(56.7)$ & $13(43.3)$ & - \\
\hline 8. & Defibrillator & $4(17.3)$ & $19(82.6)$ & 7 \\
\hline 9. & Stethoscope & $29(96.7)$ & $1(3.3)$ & - \\
\hline 10. & Pulse oximeter & $29(96.7)$ & $1(3.3)$ & - \\
\hline 11. & Extra I/V fluids, inotropic solution, or blood & $12(40)$ & $18(60)$ & - \\
\hline 12. & $\begin{array}{l}\text { Resuscitation drugs } \\
\text { (inj. epinephrine, inj. atropine, } \\
\text { inj. amiodarone with filter, inj. sodium } \\
\text { bicarbonate, and Inj. midazolam) }\end{array}$ & $21(70)$ & $9(30)$ & - \\
\hline 13. & Twist drill and brain cannula & $4(26.6)$ & $11(73.3)$ & 15 \\
\hline 14. & Torch & $16(53.3)$ & $14(46.7)$ & - \\
\hline 15. & Portable I/V stands & $27(90)$ & $3(10)$ & - \\
\hline 16. & Infusion pump & $19(63.3)$ & $11(36.7)$ & - \\
\hline 17. & Patient notes and imaging films & $30(100)$ & - & - \\
\hline
\end{tabular}

functioning of trolleys were proper in 23 (76.7\%) cases. In 19 (70.3\%) transports, intubation kit were carried during transport. Oxygen cylinder and oxygen tubing were carried in all transportation, but were not used in three cases.

In $5(20 \%)$ transports, portable cardiac monitor and in 29 (96.7\%) transports stethoscope and pulse oximeter were carried during transport. In four (26.6\%) transports, twist drill and brain cannula were taken during transport. Twenty-seven cases carried along portable I/V stands while transportation. Patient notes and imaging films were accompanied in all transports.

- Table 5 indicates that Glasgow Coma Scale of patient were checked in 29 (96.7\%) samples before transport. Temperature in 28 (93.3\%), pulse in 4 (96.7\%), respiration rate in 29 (96.7\%), and blood pressure in all samples were checked before transport. Airway was secured and checked for patency in all samples. In 29 (96.7\%) samples, all drains' functioning was checked. In 26 (86.7\%) samples, functioning of I/V drips and infusion pumps was checked. Side rails were raised in $20(66.7 \%)$ samples.

- Table 6 indicates that in all transports, clinical status of patient was documented before transport, and name of referring and receiving authority was documented in 25
(83.3\%) samples. Indication, time, and place of transport were documented in 29 (96.7\%) transports.

In none of the cases, any life-threatening adverse events happened. Half of the patients had problems of parenteral tubing blockade due to tangling during transit.

- Table 7 shows that receiving team was found to be fully prepared in 28 (93.3\%) cases and had fully equipped monitors, ventilators, and drug administration equipment along with power cords. In 24 (80\%) cases, transporting team remained alongside the receiving team till all monitoring equipment and life-support systems were put to use and patient parameters recorded.

\section{Discussion}

Radiological investigation of CT scan remains the most common cause for transporting the critically sick neurosurgical patient, as shown by our study, supporting the previous literature. ${ }^{5}$ This cause is obvious and well understandable as repeated radiological investigation of CT scan remains the primary requirement to monitor the clinico-radiological correlation for these patients and requirement of intervention thereof. 
Table 5 Pretransport patient status assessment $(N=30)$

\begin{tabular}{|c|c|c|c|c|}
\hline SI. no. & Content & Done, $n(\%)$ & Not done, $n(\%)$ & Not applicable, $n$ \\
\hline 1. & Glasgow Coma Scale documented (EVM) & $29(96.7)$ & $1(3.3)$ & - \\
\hline \multirow[t]{5}{*}{2.} & Vitals documented & & & \\
\hline & Temperature & $28(93.3)$ & $2(6.7)$ & - \\
\hline & Pulse & $29(96.7)$ & $1(3.3)$ & - \\
\hline & Respiration rate & $29(96.7)$ & $1(3.3)$ & - \\
\hline & $\mathrm{BP}$ & $30(100)$ & - & - \\
\hline \multirow[t]{3}{*}{3.} & Respiratory & & & \\
\hline & Airway secured and patency checked & $30(100)$ & - & - \\
\hline & Breath sounds assessed & $7(23.3)$ & $23(76.7)$ & - \\
\hline \multirow[t]{2}{*}{4.} & Cardiovascular & & & \\
\hline & ECG assessment for arrhythmias & $1(3.3)$ & $29(96.7)$ & - \\
\hline 5. & All drains are functioning and secured & $29(96.7)$ & $1(3.3)$ & - \\
\hline 6. & $\begin{array}{l}\text { Water seal drain clamped or not according } \\
\text { to instruction }\end{array}$ & - & $29(100)$ & 1 \\
\hline 7. & $\begin{array}{l}\text { Functioning of } \mathrm{I} / \mathrm{V} \text { drips and infusion } \\
\text { pumps checked }\end{array}$ & $26(86.7)$ & $4(13.3)$ & - \\
\hline 8. & Side rails raised & $20(66.7)$ & $10(33.3)$ & - \\
\hline
\end{tabular}

The present cause seems to be modifiable with the recent availability of portable CT scans alongside ICU and neurosurgical operation theaters. Besides the high cost, radiation exposure remains the deterrent of the availability of this facility in most of the neurosurgical centers especially in developing countries.

Only one study has reported transportation time (mean of 80 minutes), but the present study has significantly less time consumption ( 25 minutes) probably due to proximity of departing-receiving areas and well communications beforehand while shifting. As transportation time has significant bearing on incidence of technical mishaps, reducing this time should remain a priority for every neurosurgical center. ${ }^{6}$

Transportation harbors changes in biophysiological parameters which are well known in English literature. Such changes are not described in specifically neurosurgical patients. The present study showed changes in systemic

Table 6 Documentation for intrahospital transport of critically sick neurosurgical patients $(N=30)$

\begin{tabular}{|l|l|l|l|l|}
\hline Sl. no. & Content & Done, $\boldsymbol{n}(\%)$ & Not done, $\boldsymbol{n}$ (\%) & Not applicable, $\boldsymbol{n}$ \\
\hline & Clinical status of patient documented & $30(100)$ & - & - \\
\hline & Indication, time, and place of transport documented & $29(96.7)$ & $1(3.3)$ & - \\
\hline & Name of referring and receiving authority & $25(83.3)$ & $5(16.7)$ & - \\
\hline
\end{tabular}

Table 7 Arrival procedure for intrahospital transportation of critically sick neurosurgical patients $(N=30)$

\begin{tabular}{|l|l|l|l|l|}
\hline Sl. no. & Content & Done, $\boldsymbol{n}(\%)$ & Not done, $\boldsymbol{n}(\%)$ & Not applicable, $\boldsymbol{n}$ \\
\hline 1. & Receiving unit prepared to receive patient & $28(93.3)$ & $2(6.7)$ & - \\
\hline 2. & Proper body mechanics followed while shifting patient & $14(46.7)$ & $16(53.3)$ & - \\
\hline 3. & $\begin{array}{l}\text { Patient assessed when new monitors, } \\
\text { ventilators and power supplies established }\end{array}$ & $27(90)$ & $3(10)$ & - \\
\hline 4. & Complete handover given to receiving team & $24(80)$ & $6(20)$ & - \\
\hline 5. & $\begin{array}{l}\text { Transport staff remain with the patient until } \\
\text { receiving team fully take over }\end{array}$ & $24(80)$ & - \\
\hline
\end{tabular}


blood pressures, oxygen saturation, pulse rate, and neurological status, but changes in respiration rates were significant. This is probably due to shift of patients from mechanical ventilators to manually assisted mechanical breathing unit ventilation and significant number of patients harboring hospital-acquired chest infections.

The present study involved accompanying equipment as life-saving ones, which included oxygen cylinder, Assisted Mechanical Breathing Unit bag, laryngoscope, oxygen tubing, stethoscope, pulse oximeter, infusion pump, and masks of appropriate sizes. Surprisingly, literature has shown that technical mishaps are more likely with greater monitoring devices and, henceforth, curtailing of such supporting equipment should be practiced. ${ }^{6}$

The present study had technically trained health personnel accompanying all transportations, which was nursing personnel in 12 (40\%) cases, while medical health care professional was there in all cases. We did not encounter any untoward mishap or technical failure leading to any morbidity or mortality in the present study. The literature also supports the ritual of accompanying trained health care professional for such transportations, and complication of as less as just $2.8 \%$ has been reported. ${ }^{6-9}$

Protocol for intrahospital transport of critically sick neurosurgical patients helps to standardize the equipment, personnel, and monitoring required during this transit period. The quality and consistency of clinical care during transport can be enhanced by the availability of clear evidence-based guidelines. These clinical protocols are beneficial for both the treating team and patients. ${ }^{10}$

Small sample size remains the major limitation of the present study, and the influence of investigators' presence has the Hawthorne effect.

\section{Conclusion}

Transportation of neurosurgically sick patients has inherent risks of mishaps accounting from minor to major incidents, including fatalities. The established protocols displayed and accepted among health care professionals can minimize such incidents. Minimizing transportations by bringing investigative modalities at hand like portable CT scan and minimizing time of transportation will also help taking these untoward incidents to nadir. Prioritizing the patients who are on transportation for investigations and booking the transport pathways like corridor, lifts, and ramps would further minimize the transport time, thereby decreasing the unwanted incidents.

\section{References}

1 Waydhas C. Intrahospital transport of critically ill patients. Critical Care 1999;3:83-89

2 Beckmann U, Gillies DM, Berenholtz SM, Pronovost P. Incidents relating to the intra-hospital transfer of critically ill patients. An analysis of the reports submitted to the Australian Incident Monitoring Study in Intensive Care. Intensive Care Med 2004; 30(8):1579-1585

3 Waydhas C, Schneck G, Duswald KH. Deterioration of respiratory function after intra-hospital transport of critically ill surgical patients. Intensive Care Med 1995;21(10):784-789

4 Ferdinande P. Recommendations for intrahospital transport of the severely head injured patient. European Society of Intensive Care Medicine statement. Intensive Care Med 1999;25: 1441-1443

5 Hurst JM, Davis K, Johnson DJ, Branson RD, Branson PS. Cost and complications during in-hospital transport of critically ill patients: a prospective cohort study. J Trauma 1992;33: 582-585

6 Doring BL, Kerr ME, Lovasik DA, Thayer T. Factors that contribute to complications during intrahospital transport of the critically ill. J Neurosci Nurs 1999;31(2):80-86

7 Kalisch BJ, Kalisch PA, Burns SM, Kocan MJ, Prendergast V. Intrahospital transport of neuro ICU patients. J Neurosci Nurs 1995;27(2):69-77

8 Shirley PJ, Bion JF. Intra-hospital transport of critically ill patients: minimising risk. Intensive Care Med 2004;30(8): 1508-1510

9 Szem JW, Hydo LJ, Fischer E, Kapur S, Klemperer J, Barie PS. Highrisk intrahospital transport of critically ill patients: safety and outcome of the necessary "road trip". Crit Care Med 1995;23(10): 1660-1666

10 Michelle T. Mobility protocol nursing standard of care. Journal of Critical Care Nurse 2004;24:87-88 